\title{
UMA ANÁLISE DO AMBIENTE VIRTUAL DE APRENDIZAGEM: ESTUDO DAS PROPOSIÇÕES DA PLATAFORMA TIM TEC E A OFERTA DE CURSOS MOOCs PARA A QUALIFICAÇÃO DE PROFESSORES UNIVERSITÁRIOS
}

\author{
Fabiana Soares Dos Santos Poschi ${ }^{1}$ \\ Sandra Dutra Piovesan ${ }^{2}$
}

\begin{abstract}
RESUMO: O estudo versa sobre a proposta da Plataforma TIM Tec para o desenvolvimento de MOOCs para uso de profissionais da educação. Apresenta por objetivo geral analisar as propostas metodológicas observadas na adoção da plataforma TIM Tec e uso de MOOCs como ferramenta de ensino. Destaca por objetivos específicos caracterizar MOOC; conhecer as possibilidades de linguagens utilizadas no MOOCs na Plataforma TIM Tec; apontar ferramentas adequadas ao processo de ensino via MOOCs no ambiente virtual TIM Tec; verificar as metodologias de ensino predominantes no ambiente de MOOCs/ TIM Tec; investigar as vantagens de uso do ambiente virtual TIM Tec para profissionais da educação. Quanto ao método o estudo utiliza de uma pesquisa bibliográfica, de abordagem qualitativa; do tipo descritivo-exploratório, partindo da análise da Plataforma TIM Tec, na realização do Curso MOOC novas formas de aprendizagem. O estudo conclui os MOOCs são modalidades de cursos online que suportam um número massivo de alunos, quebram barreiras geográficas. A oferta de MOOCs na plataforma TIM Tec é uma oportunidade de enriquecimento de aulas presenciais e de desenvolvimento de aulas e até mesmo de um curso completo na modalidade EAD. O uso do espaço tende a facilitar as tarefas dos docentes, considerando a interação entre outros aplicativos, programas e ambientes virtuais através de uso de links externos; e que o Instituto disponibiliza espaço para o desenvolvedor guardar conteúdos no formato de arquivos, ebooks, livros textos, vídeos e outros, fazendo uso de todos os tipos de linguagens (oral, escrita, simbólica, mista; formal e informal). Os docentes podem utilizar metodologia padrão, e outras, contudo a principal metodologia utilizada é a micro aprendizagem que envolve o uso de vídeos curtos.
\end{abstract}

Palavras-chave: Vídeos Curstos. Profissionais da Educação. Propostas metodológicas. Cursos Online Aberto e Massivo.

ABSTRACT: The study deals with the proposal of the TIM Tec Platform for the development of MOOCs for use by education professionals. Its general objective is to analyze the methodological proposals observed in the adoption of the TIM Tec platform and the use of MOOCs as a teaching tool. Highlights for specific objectives to characterize MOOC; know the possibilities of languages used in MOOCs on the TIM Tec

\footnotetext{
I Professora de Educação Infantil, séries iniciais e de Língua Portuguesa na Educação de Jovens e Adultos EJA. Graduada em Letras/Espanhol e Respectivas Literaturas pelo Centro Universitário da Região da Campanha -URCAMP, pós-graduada em Mídias na Educação pela Universidade Federal de Santa Maria - UFSM, mestranda no Curso de Mestrado Acadêmico em Ensino pela Universidade Federal do Pampa -UNIPAMPA (Campus Bagé $\quad$ RS). Orcid:https://orcid.org/oooo-ooor-6939-5133. Lattes: http://lattes.cnpq.br/8577754176214206.Email: fabyysoares2or4@gmail.com.

${ }^{2}$ Doutora em Informática em Educação. Possui graduação em Ciência da Computação pela Universidade de Cruz Alta (2006), graduação em Programa Especial de Graduação e Formação de Professores pela Universidade Federal de Santa Maria, equivalente a Licenciatura em Computação (2012), Mestrado em Informática pela Universidade Federal de Santa Maria (2oII) e Doutorado em Informática na Educação pela Universidade Federal do Rio Grande do Sul (2015). Atualmente é pesquisadora da Universidade Federal de Santa Maria e da Universidade Federal do Rio Grande do Sul e professora na Universidade Federal do Pampa, curso de Engenharia de Computação e Mestrado em Ensino. Tem experiência na área de Ciência da Computação, atuando principalmente nos seguintes temas: Linguagem Formais e Autômatos, Educação à Distância, Informática na Educação e Ambientes Imersivos. Email: sandrapiovesan@gmail.com Fone: 053 999333630. https://orcid.org/oooo-00o2-3175-867X.
} 
Platform; point out appropriate tools for the teaching process via MOOCs in the TIM Tec virtual environment; verify the predominant teaching methodologies in the MOOCs/TIM Tec environment; investigate the advantages of using the TIM Tec virtual environment for education professionals. As for the method, the study uses a bibliographical research, with a qualitative approach; of the descriptive-exploratory type, based on the analysis of the TIM Tec Platform, in the realization of the MOOC Course New Ways of Learning. The study concludes that MOOCs are modalities of online courses that support a massive number of students, breaking down geographic barriers. The offer of MOOCs on the TIM Tec platform is an opportunity to enrich face-to-face classes and the development of classes and even a complete course in the distance learning modality. The use of space tends to facilitate the tasks of teachers, considering the interaction between other applications, programs and virtual environments through the use of external links; and that the Institute provides space for the developer to store content in the format of files, ebooks, textbooks, videos and others, making use of all types of languages (oral, written, symbolic, mixed, formal and informal). Teachers can use standard methodology, and others, however the main methodology used is micro learning, which involves the use of short videos.

Keywords: Videos Shorts. Education professionals. Methodological proposals. Massive Open Online Course.

\section{INTRODUÇÃO}

$\mathrm{Na}$ contemporaneidade há uma propagação de Universidades e das mais distintas organizações educacionais voltadas ao fornecimento de cursos para qualificação de diferentes públicos, inclusive para a formação docente (GÓMEZ, 2015), sendo de compromisso dos profissionais a busca de habilidades, competências tecnológicas que favoreçam tanto ao processo de ensino como de aprendizagem.

Neste contexto é imprescindível que especialmente os profissionais da educação, tenham conhecimentos respectivos a espaços, instrumentos e ferramentas que promovam a inovação do ensino tornando o aprender algo prazeroso e possível de se realizar de forma dinâmica, com maior liberdade e autonomia tanto para o aluno como para o professor. A situação pandêmica resultante da disseminação do COVIDı́g reforça a urgência de uma postura arrojada por parte dos educadores, e neste cenário surgiram os MOOCs favorecendo o ensino online (PUJOL, 2020).

Os MOOCs são Cursos Online Aberto e Massivo - Massive Open Online Course, podese dizer que implicam em "socialização do aprendizado de qualidade [...], oportunizando a formação e capacitação da população, alterando os espaços de ensinar e aprender" (BARIN; BASTOS, 2013, p.I). Esta modalidade de curso é hospedada em plataformas denominadas ambientes virtuais. Um exemplo em destaque é a Plataforma TIM Tec, iniciativa do Instituto TIM Tec, "Uma organização social sem fins lucrativos (OSCIP) fundada em 2013 pela TIM que já alcançou por meio de suas ações cerca de 500 cidades, nos 26 estados e no Distrito Federal, beneficiando mais de 700 mil pessoas" (TIM TEC, 202ra, p.I). 
Este estudo versa sobre a possibilidade de uso da Plataforma TIM Tec para o desenvolvimento de MOOCs por profissionais da educação. Em análise a plataforma busca conhecer as vantagens que docentes podem usufruir se escolher a Plataforma TIM Tec para o desenvolvimento de MOOCs.

Tendo-se por hipótese inicial que os docentes poderão fazer uso do espaço TIM Tec para disponibilizar conteúdos e materiais pedagógicos, desenvolver cursos online como estratégia de ensino de diversificados temas e no mesmo sentido enriquecer aulas presenciais, sem quaisquer custos. Utiliza de uma pesquisa bibliográfica, descritivoexploratória e uma análise de abordagem qualitativa do Portal TIM Tec na observação do Curso 'MOOC e novas formas de aprendizagem'.

Desta forma o estudo tem por objetivo geral analisar as propostas metodológicas observadas na adoção da plataforma TIM Tec e uso de MOOCs como ferramenta de ensino. Destaca por objetivos específicos caracterizar MOOC; conhecer as possibilidades de linguagens utilizadas no MOOCs na Plataforma TIM Tec; apontar ferramentas adequadas ao processo de ensino via MOOCs no ambiente virtual TIM Tec; verificar as metodologias de ensino predominantes no ambiente de MOOCs/ TIM Tec; investigar as vantagens de uso do ambiente virtual TIM Tec para profissionais da educação.

Quanto ao método o estudo utiliza de uma pesquisa bibliográfica, de abordagem qualitativa; do tipo descritivo-exploratório, partindo da análise da Plataforma TIM Tec, na realização do Curso MOOC novas formas de aprendizagem. O referencial teórico do trabalho se desenvolve em dois capítulos, sendo que o primeiro versa sobre MOOCs, plataformas e formação docente. O segundo trata de uma análise da Plataforma TIM Tec, na execução do Curso 'MOOC novas formas de aprendizagem', de onde apresenta-se a disposição dos conteúdos (estruturação do Curso MOOC), os recursos utilizados e os conteúdos propriamente dito, trazendo uma discussão sobre os mesmos no confronto entre o material teórico e os achados. Observa-se que o conteúdo de análise faz parte de um capítulo de uma dissertação de mestrado em desenvolvimento (não defendida até o presente momento).

\section{Os MOOCs, Platormas e a formação docente}

Diante da disseminação de tecnologias de informação na contemporaneidade se absorveu uma cultura digital, que emergiu especialmente devido às múltiplas interações que, em um movimento contínuo se estabelecem dia após dia em um contexto de rede, traduzindo-se em movimento denominado conectivismo de onde visualiza-se o surgimento 
de um "cenário de sociedade em rede", (SILVA; MARQUES, 2015). Dentro desta realidade as TICs consolidaram-se

[...] como um importante coadjuvante no processo de aprendizagem, pois seus recursos potencializam o acesso a informações, a comunicação entre professor e aluno, o apoio a compreensão de temas complexos por meio de simuladores e aplicativos, a oportunidade de interação e colaboração entre alunos e professores são algumas possibilidades, entre muitas outras. (SÉRIE EDUCAR, 2020, p.9).

Como uma inovação do cenário educacional, surgiram os MOOCs, definidos inicialmente como um fenômeno na área de ensino, que surge na web como uma possibilidade de democratização do conhecimento, uma ferramenta de capacitação que vem favorecendo a milhares de pessoas. (BASTOS; BIAGIOTTI, 2014).

Os Cursos Abertos e Massivos, ou Moocs tiveram início aproximadamente em 2008, derivado do trabalho dos canadenses Stephen Downes e George Siemens que ofertaram um curso aberto online e gratuito denominado Conectivismo e Conhecimento Conjuntivo, de onde se instaurou um processo de oferta de "um novo modelo de aprendizagem" (ALMEIDA; MARQUES. 2015 p.3).

Desta forma diz-se que os MOOCs são hospedados em ambientes virtuais que por sua vez são delineados, ou desenhados considerando um conhecimento pedagógico, uma estrutura de ensino, ou ainda organização de conteúdos de forma sistêmica o que os caracteriza como ambientes de aprendizagem. As plataformas que ofertam MOOCs usam ferramentas da web 2.o, contudo suportam alocação de materiais em nuvem e preconizam o acesso para fins de capacitação profissional de um número massivo de inscritos. (SILVA, 2017).

O conceito admite uma concepção contemporânea que surge na área de ensino, e que vem ganhando força na formalidade de educação a distância - EaD. Todavia intencionalidade de um MOOC é disponibilizar acesso aberto, baseado em uma modalidade de educação à distância promovendo a participação interativa em grande escala apresentando conteúdos com qualidade, o que envolve organização didática, estruturação de conteúdos e subsequentes análises dos cursos depois de desenvolvidos, com feedbacks contínuos (BASTOS, 2016).

Para alcançar os propósitos do MOOC em fornecimento de qualidade de ensino, é preciso que tanto professor como aluno tenham competências básicas para usar plataformas e suas respectivas ferramentas, é preciso que haja coerência entre conteúdos fornecidos a 
distância e o presencial; que se tenha conhecimento de recursos de análise e avaliação de aprendizagem. Por fim necessário é que potencialize a aprendizagem no uso de tecnologias ativas, isto refere que "apostilas e vídeos devem ser dinâmicos e interligados. Deve-se utilizar pré e pós testes, jogos, fóruns, chats e redes sociais, ou seja os professores devem usar ferramentas interativas" (BASTOS; BIAGIOTTI, 2013, p.7 apud BASTOS, 2016 p. 52) também, o docente deve ter o cuidado de utilizar recursos educacionais abertos.

\section{Análise de 'MOOC e novas Formas de Aprendizagem': Plataforma TIM Tec}

Em análise a Plataforma TIM Tec ingressou-se no Curso "MOOC e novas formas de aprendizagem" (ver Figura I). Observou-se, inicialmente o cuidado na elaboração de cada fase respectiva a seleção ingresso e, apresentação do curso, começando pela primeira imagem de apresentação do curso, onde se observa o uso de linguagem mista também conhecida como linguagem hibrida que refere ao uso combinado das linguagens verbal (nesta fase, a escrita) e não verbal (por símbolos = imagens), com apresentação subsequente do nome do curso, os nomes dos instrutores e o tema.

Figura I - Primeira Interface: MOOC e novas formas de aprendizagem/ TIM Tec

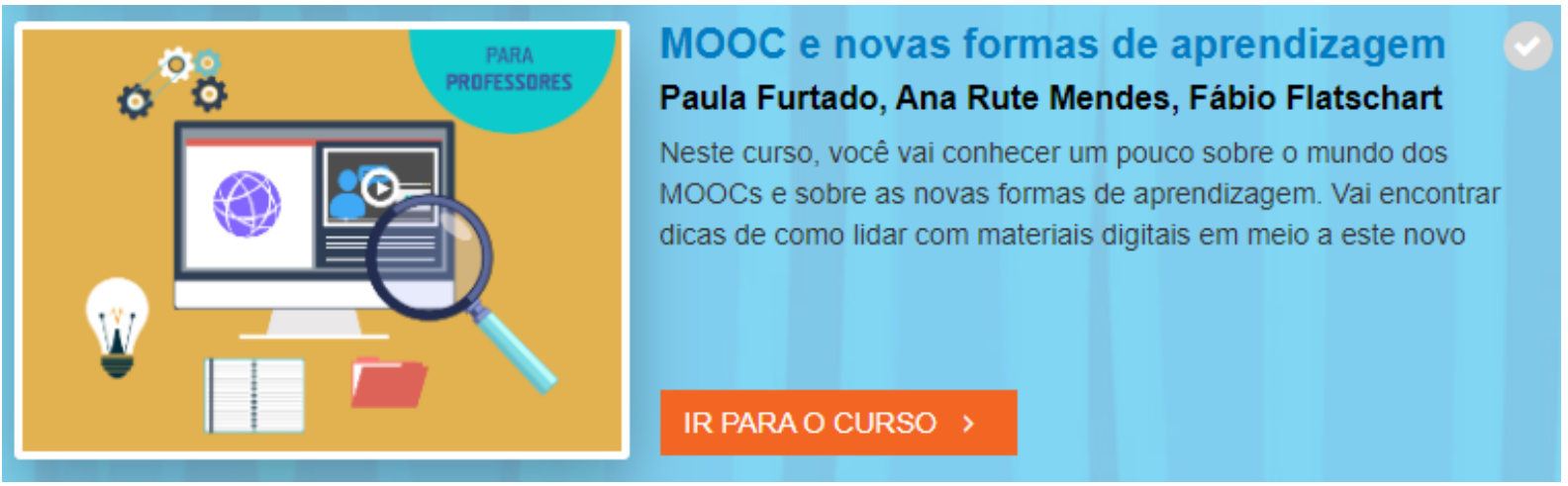

Fonte: Entrada da Plataforma TIM Tec (2021).

A seguir, ingressando no Curso, o aluno é levado à outra interface, onde se apresentam os instrutores através de um vídeo curto (Figura 2); os mesmos introduzem os principais objetivos do MOOC, usando um breve diálogo; uma síntese bem elaborada. Para o desenvolvimento do vídeo é usado o site do You Tube, assim a interação entre os ambientes TIM Tec e You Tube ocorre no uso de link externo, mas a apresentação do vídeo ocorre dentro do contexto da plataforma, como que acoplada (com a possibilidade de o usuário executar o vídeo diretamente do You Tube. 
Figura 2 - Interfaces de apresentação versus ingresso ao curso: MOOC TIM Tec

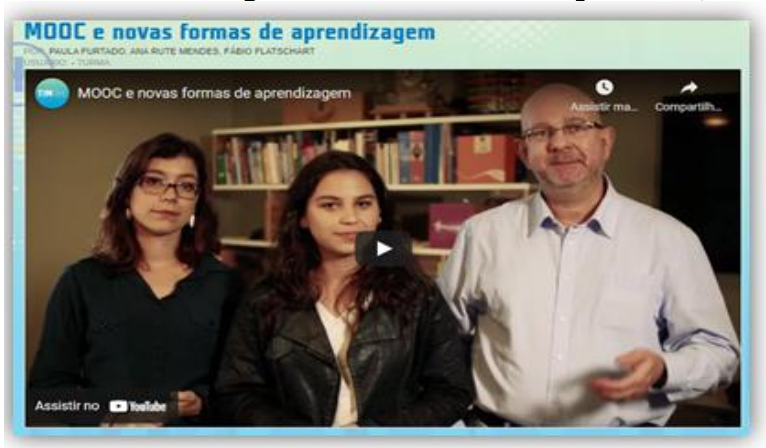

MODC e novas formas de aprendizagem

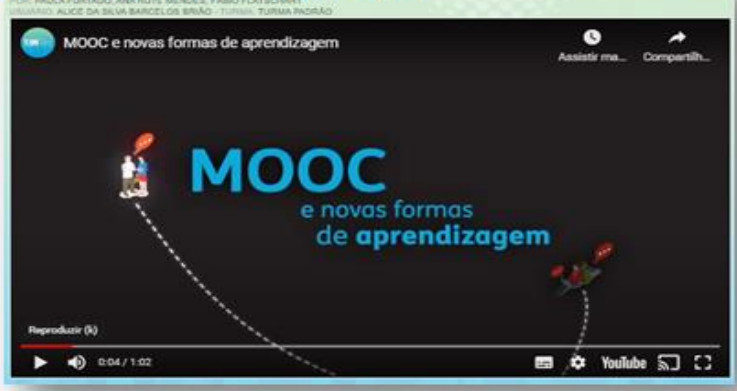

Fonte: Plataforma TIM Tec

Dentre os detalhes da interface de entrada (que apresenta um vídeo curto) observase a direita um resumo do curso, na mesma coluna um breve currículo de cada instrutor (com foto); abaixo especificações sobre o curso e, em sequencia (a baixo) a exposição das aulas observando os instrumentos utilizados.

gura 3 Descrição e estrutura do Curso (design).

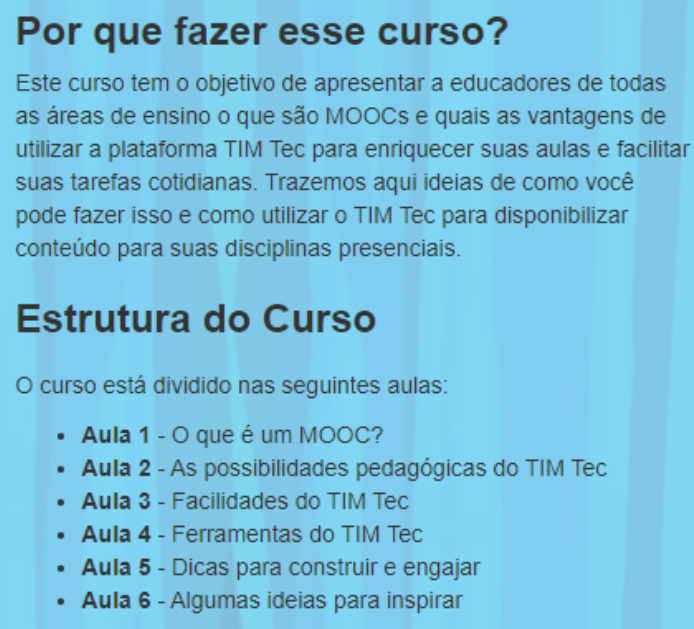

\section{Estrutura do Curso}

O curso está dividido nas seguintes aulas:

- Aula 1 - O que é um MOOC?

- Aula 2 - As possibilidades pedagógicas do TIM TeC

- Aula 3 - Facilidades do TIM Tec

- Aula 4 - Ferramentas do TIM Tec

- Aula 5 - Dicas para construir e engajar

- Aula 6 - Algumas ideias para inspirar

\section{O que eu preciso saber?}

Conhecimentos básicos de informática e internet. Nivel de dificuldade: Iniciante.

\section{Fonte: TIM Tec (202I).}

Assim, compreende-se que o Curso "MOOC e novas formas de aprendizagem" é ministrado pelos professores Paula Furtado e Ana Rute Mendes, ambas co-fundadoras da Mupi, Startup educacional de Campinas que utiliza a plataforma TIM Tec para oferecer cursos online de formação para professores. O curso também conta com o professor Fábio Flatschart, que é autor do livro HTML5 Embarque Imediato, uma das primeiras publicações em português, coautor do livro Open Web PlatForm e outras referências, o mesmo é professor 
convidado dos mais importantes cursos de Mestrado em Administração de Negócios do Brasil.

Em geral o conteúdo é apresentado em micro vídeos, sendo que cada aula é composta de um conjunto de vídeos curtos, a proposta observada refere-se a tornar dinâmicas as aulas e, o uso de linguagem informal favorece que os conteúdos sejam significativos para o aluno.

Quanto à estrutura do curso da TIM Tec:

- O curso é dividido em aulas; como se observa na Figura 3, e na Tabela I, divide-se em 6 aulas;

- Cada aula é formada por uma sequencia de capítulos (cada aula tem um jogo de capítulos), assim as aulas apresentam temas e os capítulos subtemas.

Conforme esquema de estudo das aulas, observados na Tabela I, a aula or - primeira aula, com tema "o que é um MOOC?”, apresenta cinco capítulos: o primeiro capítulo apresenta como subtema 'breve panorama sobre tecnologia e educação', e utiliza como recurso um vídeo, com o tempo de execução igual a Imin44s; o segundo capítulo apresenta o subtema 'você sabe o que é um MOOC?' da mesma forma utiliza um vídeo curto como recurso (de 55s); o segundo capítulo a aula leva o subtema "como surgiram os MOOCs?" utiliza vídeos de Imin35s; o quarto capítulo utiliza vídeo e imagem PNG, o vídeo é de 5s (só introdutório), e trata do subtema refere a "aprofundando-se nos MOOCs" e o último capítulo leva por subtema "conheça alguns exemplos de MOOCs" utiliza introdução por vídeo curto de 5s, e conteúdo expositivo em texto.

Tabela I Esquema de estudo das aulas: MOOC novas formas de aprendizagem / TIM Tec.

\begin{tabular}{|c|c|c|c|c|}
\hline AULAS / TEMAS & Capít, & SUB-TEMAS & RECURSOS & Temp. \\
\hline \multirow{6}{*}{$\begin{array}{c}\text { AULA or } \\
\text { O que é um MOOC? }\end{array}$} & I & $\begin{array}{l}\text { Breve Panorama sobre } \\
\text { Tecnologia e Educação }\end{array}$ & VÍDEO & or:44 \\
\hline & 2 & Você sabe o que é um MOOC? & VÍDEO & $00: 52$ \\
\hline & 3 & Como surgiram os MOOCs? & VÍDEO & oI:38 \\
\hline & 4 & Aprofundando-se nos MOOCs & VÍDEO e imagem PNG & $00: 05$ \\
\hline & 5 & $\begin{array}{l}\text { Conheça alguns exemplos de } \\
\text { MOOCs }\end{array}$ & $\begin{array}{l}\text { VÍDEO e aula expositiva em } \\
\text { texto }\end{array}$ & 00:05 \\
\hline & Capít, & SUB-TEMAS & RECURSOS & Temp. \\
\hline \multirow[t]{2}{*}{ AULA 02} & \multirow[t]{2}{*}{ I } & Muito mais que MOOCs & VÍDEO & 01:12 \\
\hline & & $\begin{array}{l}\text { Atividades complementares: } \\
\text { expandindo os horizontes }\end{array}$ & VÍDEO & OI:20 \\
\hline $\begin{array}{c}\text { As possibilidades } \\
\text { pedagógicas do TIM } \\
\text { Tec }\end{array}$ & 3 & $\begin{array}{l}\text { Atividades de reforço: } \\
\text { diferentes caminhos de } \\
\text { aprendizagem }\end{array}$ & VÍDEO & 02:10 \\
\hline
\end{tabular}




\begin{tabular}{|c|c|c|c|c|}
\hline & \multirow[t]{2}{*}{4} & Reposição de aula & VÍDEO & or:10 \\
\hline & & $\begin{array}{l}\text { Desenvolvimento do projeto } \\
\text { pedagógico }\end{array}$ & VÍDEO & OI:31 \\
\hline \multirow{4}{*}{$\begin{array}{c}\text { Facilidades da TIM } \\
\text { Tec }\end{array}$} & Capít, & SUB-TEMAS & RECURSOS & Temp. \\
\hline & I & $\begin{array}{l}\text { Atualização rápida e prática de } \\
\text { materiais }\end{array}$ & VÍDEO & $02: 15$ \\
\hline & 2 & $\begin{array}{l}\text { Conexão, reutilização e } \\
\text { motivação }\end{array}$ & VÍDEO & $02: 14$ \\
\hline & 3 & $\begin{array}{l}\text { Você mais próximo da sua } \\
\text { turma }\end{array}$ & VÍDEO & 01:02 \\
\hline \multirow{5}{*}{$\begin{array}{c}\text { Ferramentas do TIM } \\
\text { Tec }\end{array}$} & Capít, & SUB-TEMAS & RECURSOS & Temp. \\
\hline & I & TIM Tec na prática & Vídeo introdut. & $00: 23$ \\
\hline & 2 & Tipos de atividades disponíveis & $\begin{array}{l}\text { Aula expositiva, texto e gif } \\
\text { animado }\end{array}$ & \\
\hline & 3 & Os papéis de usuário & $\begin{array}{l}\text { Aula expositiva, texto e gif } \\
\text { animado }\end{array}$ & \\
\hline & 4 & Gestão de turmas & $\begin{array}{l}\text { Aula expositiva, texto e gif } \\
\text { animado }\end{array}$ & \\
\hline \multirow{10}{*}{$\begin{array}{l}\text { Dicas para Construir } \\
\text { e Engajar }\end{array}$} & Capít, & SUB-TEMAS & RECURSOS & Temp. \\
\hline & I & $\begin{array}{l}\text { Dicas para produção de } \\
\text { conteúdo }\end{array}$ & Vídeo introdutório & $00: 48$ \\
\hline & 2 & Introdução & Introdução em texto & \\
\hline & 3 & Design Instrucional & Aula expositiva em texto & \\
\hline & 4 & Dicas para planejamento & $\begin{array}{l}\text { Aula expositiva, texto em } \\
\text { quadros }\end{array}$ & \\
\hline & 5 & $\begin{array}{l}\text { Dicas para desenvolvimento de } \\
\text { conteúdos }\end{array}$ & $\begin{array}{l}\text { Aula expositiva, texto em } \\
\text { quadros }\end{array}$ & \\
\hline & 6 & Autoria e reutilização na Web & $\begin{array}{l}\text { Aula expositiva, texto e } \\
\text { imagem }\end{array}$ & \\
\hline & 7 & $\begin{array}{l}\text { Dicas para produzir seus } \\
\text { próprios vídeos }\end{array}$ & $\begin{array}{l}\text { Aula espositiva, texto e } \\
\text { quadros }\end{array}$ & \\
\hline & 8 & $\begin{array}{l}\text { Mais dicas para produzir seus } \\
\text { próprios vídeos }\end{array}$ & $\begin{array}{l}\text { Aula expositiva, texto e } \\
\text { imagem }\end{array}$ & \\
\hline & 9 & Sequência de aprendizagem & Aula expositiva em texto & \\
\hline \multirow{9}{*}{$\begin{array}{c}\text { Algumas ideias para } \\
\text { inspirar }\end{array}$} & Capít, & SUB-TEMAS & RECURSOS & Temp. \\
\hline & I & Ideias & Expositiva com imagem e texto & \\
\hline & 2 & Vídeos de fabricantes & Expositiva com texto & \\
\hline & 3 & Setup e procedimento & $\begin{array}{l}\text { Expositiva texto em quadros e } \\
\text { imagens }\end{array}$ & \\
\hline & 4 & $\begin{array}{l}\text { Apresentação de software e } \\
\text { ferramentas }\end{array}$ & $\begin{array}{l}\text { Expositiva com texto/ link } \\
\text { externo para download } \\
\text { programas }\end{array}$ & \\
\hline & 5 & $\begin{array}{l}\text { Atividades e conteúdos para } \\
\text { reforço }\end{array}$ & Expositiva com texto & \\
\hline & 6 & Vídeos aulas com voz e slides & Expositiva com texto & \\
\hline & 7 & $\begin{array}{l}\text { Não se limite ao espaço de sala } \\
\text { de aula }\end{array}$ & Expositiva texto em quadros & \\
\hline & 8 & Encerramento & Vídeo de encerramento. & oo:37 \\
\hline
\end{tabular}

Fonte: a autora (2021). 
Assim, na primeira aula, no capítulo I, o primeiro vídeo de ormin44s, discorrendo sobre o subtema 'breve panorama sobre tecnologia e educação', Ana Rute Mendes, explica de forma natural a importância de se conjugar tecnologia à educação, mesmo por que as tecnologias inegavelmente fazem parte do dia-a-dia das pessoas, ou seja quase tudo funciona no uso das tecnologias, o que deve ser levando em conta nas formas de ensinar para ganhar a motivação dos alunos para o aprender. Mendes (202I) coloca que "por o meio das tecnologias, se por um lado a gente aumenta a nossa capacidade de distribuir conteúdos e informação, por outro lado a gente tem um público maior, cada vez mais heterogêneo entre si”, e sobre esta realidade reconhece o desafio de desenvolver curso para um número massivo de gente, e a natureza dos conteúdos a serem ensinados,

Mendes (202I) refere sobre a importância de compreendermos e revermos o conceito de MOOCs, sabendo os seus fins; pois há um grande desafio no planejamento deste tipo de curso relacionado à heterogeneidade e a quantidade dos públicos que se deseja alcançar, de forma que realmente estes públicos tenham um bom aceite (sejam envolvidos e engajados) com excelente produtividade, ou seja, resultados de aprendizagem, qualificada. Assim a primeira proposta dos MOOCs, considerada pela instrutora TIM Tec, é "usar as tecnologias como nossas aliadas e aumentar as possibilidades de oferecer, cursos e conteúdos de qualidade para um maior número de pessoas".

O panorama tecnologia educação vem apresentando continuas modificações, fenômenos como o conectivismo, que refere à interação em rede, desencadearam a assimilação de uma cultura digital e os MOOCs foram surgiram como um fenômeno de resposta a "necessidade da criação de ferramentas de ensino" (BASTOS; BIAGIOTTI, 2014), tais como a necessidade de educação continuada - lifelong learning em ambientes corporativos, de se formar redes de aprendizagem e de democratização do ensino.

Neste cenário as tecnologias são entendidas como:

[...] fortes aliadas dos professores no processo de ensino-aprendizagem [...], porém o uso de recursos tecnológicos exige dos professores constantes atualizações. Sendo assim, é importante, no cenário brasileiro, identificar quais ferramentas computacionais estão disponíveis de forma gratuita na formação dos professores para capacitá-los/auxiliá-los [...].

Ambientes $\mathrm{EaD}$ são uma boa opção, e, dentro dessa área os MOOCs são os que oferecem acesso gratuito e com flexibilidade de horário para que se possa realizar 
uma capacitação centrada na disponibilidade de tempo do professor (BALBINO; PINTO; BRAZ; 2020, p.181-I82)

A globalização implicou em descentralização do conhecimento e desencadeou uma disseminação de novas tecnologias que invadiram o cenário mercadológico inserindo uma variação de Tecnologias de Informação e Comunicação - TICs, gerando novos hábitos na rotina de pessoas, nos lares, e nos diferentes contextos (de diversão, trabalho, e educação, entre outros), de forma que não dá para se viver sem elas, pois se vive uma era digital, e admite-se uma cultura digital. É preciso que docentes apropriem-se dos artefatos tecnológicos. Diante das articulações apresentadas compreende-se que sendo os Cursos MOOCs uma inovação tecnológica voltada à qualificação do processo de ensino, ofertadas de forma gratuita, se traduzem em ferramenta apropriada para a qualificação docente, e também aliada (do docente) por gerar novas e diversificadas possibilidades pedagógicas, de forma que os professores podem ofertar cursos sobre conteúdos específicos, qualificando ou potencializando o ensino.

Ainda na primeira aula, segundo vídeo, de 52s, o Capítulo 2 desenvolve-se sob o tema 'Você sabe o que é um MOOC?'. O Professor Fábio Flatschart introduz o tema MOOC. Traz uma questão norteadora da abordagem: "MOOC é uma plataforma ou uma nova maneira de ensinar? afinal o que é um MOOC?" (FLATSCHART, 202I) Esclarece que o MOOC é uma sigla, um Curso Online Aberto e Massivo, e não uma plataforma. Salienta que mais adiante vai se falar na plataforma TIM Tec e suas "possibilidades de apoio pedagógico que ela pode oferecer" (FLATSCHART, 202I).

No Capítulo 3, encontra-se o terceiro vídeo de ormin38s, com a abordagem refere a 'como surgiram os MOOCs': com Paula Furtado. Muito embora a professora tenha por foco observar como surgiram os MOOCs o faz de forma natural, com suas palavras, utiliza em um só momento o ano em que surgiram os MOOCs, sem fazer leitura de texto, naturalmente demonstrando um empoderamento. Diante da evidência de que há múltiplos dispositivos interconectados, que compartilham de aplicativos e programas e ambientes e redes sociais. Ela observa os avanços computacionais como possibilidade de acesso a conteúdos e a conexão entre pessoas. Questiona:

Porque não pensar em ampliar as condições de formação, e já que temos tecnologias para isto por que não pensar nisto considerando o acesso para milhares de pessoas". [...] que tal fazer parte desse movimento e ampliar também a sua sala de aula. (FURTADO, 202I). 
No capítulo 4, sob o tema: 'Aprofundando-se nos MOOCs'. É usado um tipo de mapa mental para apresentar o conceito de MOOC e ligar esta modalidade de curso a plataforma TIM Tec.

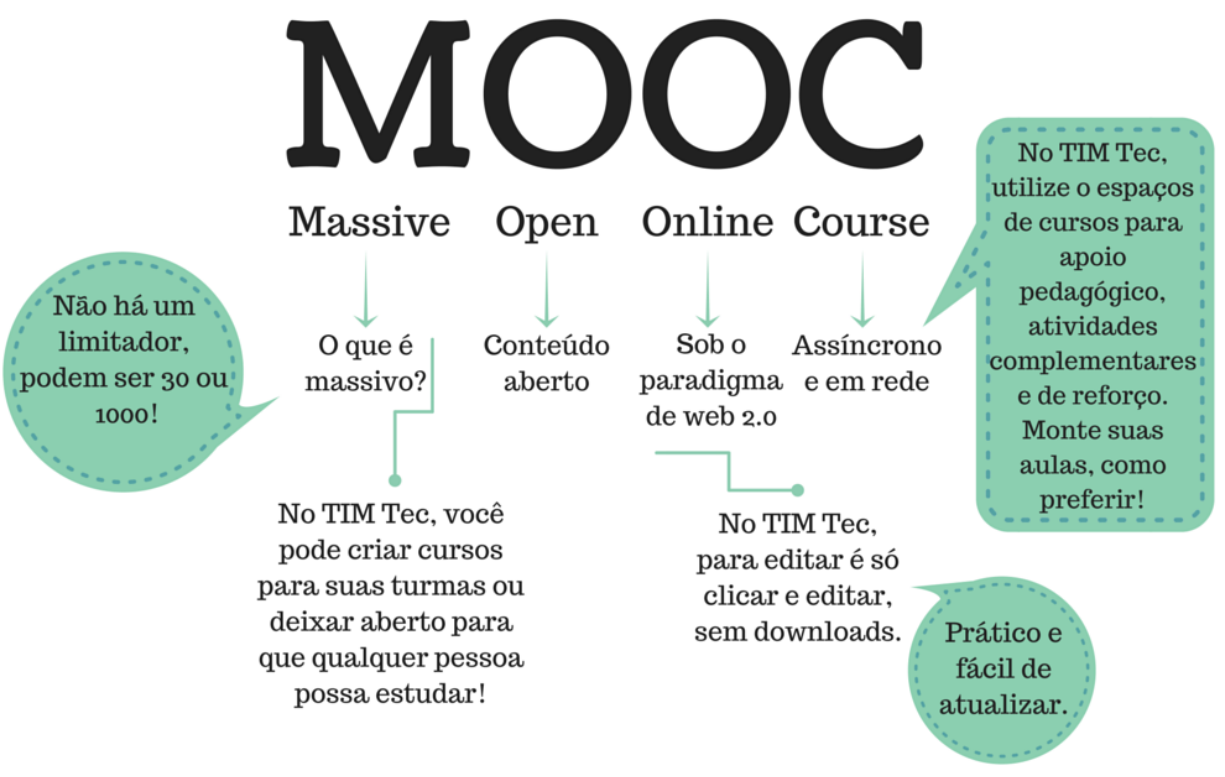

Fonte: TIM Tec (2021).

$\mathrm{Na}$ imagem há uma orientação a que se use o 'espaço de cursos para apoio pedagógico, atividades complementares e de reforço' e encerra observando de forma direta que os MOOCs são ferramentas de suporte pedagógico e o contexto das plataformas TIM Tec é adequado para montagem de aulas, "prático e fácil de atualizar" (TIM_TEC, 202I).

Os Cursos Abertos e Massivos, denominados MOOCs referidos por o instrutor, tiveram seu inicio em 2008, e são resultado do trabalho dos canadenses Stephen Downes e George Siemens surgindo como uma nova forma de ensino, a ideia central dos mesmos referia a democratizar o conhecimento, gerando uma forma coletiva de aprendizagem, acelerando assim o processo evolutivo, tendo um aproveitamento do fenômeno conectivismo, que refere as integrações em rede (ALMEIDA; MARQUES. 2015).

Conclui-se sobre a abordagem, que era necessário e, inevitável à introdução de cursos deste gênero visto a acirrada evolução das tecnologias, e o envolvimento a motivação das 
pessoas na assimilação de uso destas, de forma que as organizações educacionais são impulsionadas a aceitação dos MOOCs, a título de ficarem obsoletas, é preciso agregar aos métodos e práticas educacionais conceitos contemporâneos, e entrar no ritmo da evolução.

O Capítulo 5 inicia como se fossemos entrar em um novo vídeo, contudo apresenta um texto, sobre os exemplos de MOOCs, apontando as características de algumas plataformas.

A Coursera é uma plataforma utilizada para universidades ofertarem cursos, que podem ou não oferecer certificado, se o aluno assim o quiser deverá pagar uma taxa que se diferencia de curso para curso. A edX também disponibiliza cursos ofertados por universidades de renome contudo apresenta espaço para qualquer pessoa criar seu próprio MOOC. A TIM Tec observa que oferece MOOCs, mas não refere que se tem a liberdade de usar a plataforma para a realização de um curso próprio. E a Udacity "tem cursos voltados para formação em tecnologia".

O curso apresenta abaixo de cada vídeo, imagem ou texto que refere ao conteúdo do capítulo um espaço em que o curso pode ou não disponibilizar materiais adicionais e em seguida um fórum, que parece único disponível a todos os capítulos.

Os MOOCs são alocados em espaços virtuais, denominados plataformas de ensino, ou de ambientes virtuais de aprendizagem, em geral ofertados por renomeadas universidades, Os ambientes virtuais podem ser do tipo MOODLE, AVA, que se utilizam do Web. A Plataforma TIM Tec, é uma iniciativa da empresa de Telefonia TIM Tec, ofertada pelo Instituto TIM como uma ação social, ofertando parceria com a Programa Nacional de Acesso ao Ensino Técnico e Emprego - PRONATEC. Estes ambientes incorporam recursos e apresentam características que facilitam a gestão da aprendizagem. Conforme observou-se estes recursos são ofertados conforme demanda e tendo ligação com os avanços tecnológicos recebem atualizações. Considera importante a definição de quem poderá ofertar os cursos, pois este fato atenta para a qualidade do ensino, a restrição à docentes, organizações educacionais e até mesmo da área de abrangência é um quesito que pode contar para o enriquecimento do currículo do participante/aluno. Ou seja, os MOOCs alcançam um número massivo de pessoas, quanto alunos, mas as plataformas em geral estão disponíveis a professores, universidades, e podem ser disponibilizadas a corporações. 
Para a aula 02 é utilizado uma abordagem respectiva às possibilidades pedagógicas do TIM, e para todos os capítulos são utilizados como recurso vídeos curtos; tem-se por subtemas respectivos aos capítulos, de forma subsequente: Capítulo I = 'Muito mais que MOOCs, vídeo de orminizs; Capítulo 2 = "Atividades complementares: expandindo os horizontes", apresentada por vídeo orminzos; Capítulo 3 = "Atividades de reforço: diferentes caminhos de aprendizagem", utiliza um vídeo de ozminıos; Capítulo 4 = "Reposição de aula", vídeo curto de orminios; e Capítulo 5 = "Desenvolvimento do projeto pedagógico", vídeo curto de orminzos. Como se observa no esquema de aula na tabela I.

No primeiro vídeo da aula 2, apresentado em um tempo de ormini2s, desenvolvido sob o subtema 'Possibilidades pedagógicas do TIM Tec: conheça a plataforma TIM Tec e como ela pode enriquecer sua sala de aula', com Ana Rute Mendes.

Conforme Mendes (202I) a TIM Tec desenvolveu sua plataforma com o intuito de atender as necessidades das universidades, tratando-se por tanto de uma forma de inovar, tornar mais dinâmicas e criativas as aulas e os alunos mais autônomos no seu processo de aquisição de conhecimentos. Verifica Mendes (2021) que as mudanças externas, do mundo em geral (em específico aceleradas pela disseminação de novas tecnologias) devem ser observadas, a educação precisa adequar-se (alinhar-se) a estas mudanças, acompanhar os avanços. A educação online tem admitido diferentes plataformas, e para cada tempo os ambientes apresentam ferramentas distintas, que estão ligadas a seu momento histórico ou sua realidade.

Também os objetivos das ferramentas são ligados a especificações dos diferentes cenários sociais. A TIM Tec e demais MOOCs desenvolvem materiais e ferramentas, didáticas com foco em desenvolver habilidades e competências (autonomia, capacidade de selecionar conteúdos, colaboração e conexão).

Explica Mendes (202I) que a TIM Tec busca ir um pouco mais além "pode usar os seus recursos pra trabalhar as novas competências dos estudantes de forma independentes e criar novas possibilidades de aulas" (específico aceleradas pela disseminação de novas tecnologias) devem ser observadas, a educação precisa adequar-se (alinhar-se) a estas mudanças, acompanhar os avanços. A educação online tem admitido diferentes plataformas, e para cada tempo os ambientes apresentam ferramentas distintas, que estão ligadas a seu momento histórico ou sua realidade. 
Ainda na aula 2, no Capítulo 2, segundo vídeo de orminzos, Prof. Fábio Flatschart fala sobre 'atividades complementares: expandindo os horizontes'.

Em sua introdução reconhece que as atividades complementares são comuns nos diferentes contextos educacionais, e nesta perspectiva entende o desafio de desenvolvimento destas atividades. Orienta o docente ao uso da plataforma TIM Tec para desenvolver as atividades complementares de diferentes formas, inclusive como uma sequência didática, $e$ realça que a comunicação é assíncrona, os conteúdos ficam no ambiente podendo ser adaptados a públicos distintos, também refere que é fácil a atualização de conteúdos. E a plataforma favorece acompanhamento (do progresso) da produção, do comportamento, e dificuldades dos alunos.

$\mathrm{Na}$ aula 2, no Capitulo 3, no terceiro vídeo, desenvolvido em um tempo de ozminios, sob o subtema 'Atividades de Reforço: diferentes caminhos de aprendizagem', com Paula Furtado.

Nesta abordagem, Furtado (2021) aponta as diferentes características dos alunos, a subjetividade deste público (no MOOCs muito mais heterogêneos, com maiores demandas) todos tem Formas, tempos diferentes de aprendizagem. "cada um tem o seu próprio estilo de aprendizagem, e o seu próprio estilo cognitivo. Uns são mais auditivos, outros são mais visuais, e já outros se sentem mais confortáveis da maneira tradicional, que é aprender por meio da leitura e escrita" (FURTADO, 202I).

Salienta também o aspecto referente às velocidades de aprendizagem, destaca que há consciência dos diferentes fatores que alteram este aspecto, com destaque realça o "repertório de conhecimento do aluno e o seu ritmo de aprendizagem" (FURTADO, 202I).

Neste sentido orienta ao uso da plataforma TIM Tec para o desenvolvimento de atividades com as características, ou finalidade de material de reforço. Neste aspecto observa-se que podem ser analisados os alunos e serem adequados conteúdos (novamente) tomando por fundamento as diferentes demandas de cada aluno. A docente exemplifica apontando três diferentes tipos de recursos que podem ser usados (tipos de aulas).

$\mathrm{Na}$ aula 2, Capítulo 4, o quarto vídeo de orminios trata sobre a 'reposição de aula', com Ana Rute Mendes. Mendes (2021) introduz a temática da reposição de aulas presenciais, de forma que sugere o uso da plataforma TIM Tec para estes fins, disponibilizando o "conteúdo da aula perdida de forma online", de forma que os alunos poderão rever esta aula 
quantas vezes forem necessárias. Sugere o uso do webcam do celular, tablete ou computador; e que o professor ouse apresentar conteúdos como se estivesse conversando com os alunos. Dentro deste formato de aula, podem ser inseridos textos e outros materiais "recheando a aula", e os alunos podem acessar conteúdos no tempo disponível e em qualquer lugar que estiver e interagir com os demais alunos nos fóruns.

A aula 2, no Capítulo 5, encontra-se o quinto vídeo com orminzıs que versa sobre "desenvolvimento do projeto acadêmico", com Fábio Flatschart. Neste capítulo Flatschart. observa sobre a possibilidade de uso da plataforma TIM Tec para comunicação e interação entre aluno e docente. Neste contexto ele aponta que os docentes podem disponibilizar materiais, manuais, tutoriais para os alunos criarem seus próprios projetos. Específica por exemplo que o docente pode deixar disponível:

Diretrizes de criação de um projeto; Materiais de apoio em diferentes formatos; como slides, documentos e pdfs para conteúdos como regras da ABNT, cheklist ou, outros elementos para ajudar os estudantes a se organizarem durante suas pesquisas, entre outras aplicações.

Como um canal direto de comunicação os estudantes podem criar tópicos no fórum para tirar duvidas entre si e com você.

Além disso, a ferramenta de mensagens permite que você envie e-mails por turmas, o que torna possível o envio de lembrete sobre prazos, dicas, em fim qualquer mensagem que você queira passar a seus estudantes. Isto tudo pode ser feito utilizando a ferramenta de criação de cursos do TIM Tec, Você pode se apropriar desta ferramenta como lhe for mais conveniente, ou seja, você pode usá-la para criar um espaço para uma disciplina em que você coloca materiais adicionais, em que os estudantes podem colocar suas dúvidas. O que não precisa necessariamente ser um curso. É você quem manda! (FLATSCHART, 202I).

O tema abordado na aula 03 refere a "Facilidades da TIM Tec", desenvolve-se em três capítulos sendo que: o Capítulo or trata do subtema "atualização rápida e prática de materiais" para tanto, utiliza um micro vídeo de o2minıss.; O Capítulo o2 versa sobre “conexão, reutilização e motivação”, para apresentação do conteúdo utiliza um vídeo curto de o2minı4s.; e encerra com o Capítulo 03 com o subtema "você mais próximo da sua turma" apresentado também por micro vídeo de ormin:ozs.

Para o desenvolvimento da aula 4, que trata do tema "Ferramentas do TIM Tec", os desenvolvedores utilizaram-se de quatro capítulos, sendo que: o Capítulo or versa sobre o “TIM Tec na prática”, iniciando com um vídeo de oo:23s. e a seguir, segue ao Capítulo o2 que utiliza de uma aula expositiva em texto (tipos de atividades disponíveis) com acompanhamento de um gif animado; no Capítulo o3 é tratado o tema "os papéis de usuário", 
e o Capítulo o4 que trata sobre "gestão de turmas", ambos fazem uso de exposição de conteúdo em texto e gif animado.

Para a aula 05, desenvolvida sob o tema "Dicas para Construir e Engajar", os desenvolvedores utilizaram-se de nove capítulos, que versam sobre "Dicas para a produção de conteúdos”, "Introdução”, "Design Instrucional”, "Dicas para planejamento”, "Dicas para desenvolvimento de conteúdos”, “Autoria e reutilização na Web”, "Dicas para produzir seus próprios vídeos", "Mais dicas para produzir seus próprios vídeos”, "sequência de aprendizagem", para apresentação dos conteúdos utilizam-se de um vídeo curto introdutório de oo:48s., e aulas expositivas textuais, textuais em quadros, e textuais com imagens.

Para o desenvolvimento da ultima aula, a aula o6, desenvolvida sob o título "Algumas ideias para inspirar", os desenvolvedores utilizaram-se de oito capítulos, com o uso de aulas expositivas, com imagem e texto, expositivas só com texto, expositivas com textos quadros e imagens, expositiva com texto e link externo para download de programas; e vídeo de encerramento.

\section{CONSIDERAÇÕES FINAIS}

Em análise ao Curso 'MOOC novas formas de aprendizagem', observou-se que para a apresentação de conteúdos os docentes podem utilizar materiais com linguagem escrita, oral e visual, ou ainda hibrida. Tem opção de apresentar aulas expositivas em textos (textos editados diretamente na plataforma, ou em arquivos com extensão do tipo PDFs, Doc entre outros), mistas (no uso de figuras, símbolos, mapas mentais, slides entre outros recursos) ou expositivas orais em vídeos. Utiliza a estruturação adequada de conteúdos, o uso de temas, e subtemas para melhor sistematização do conhecimento.

A TIM Tec na apresentação do vídeo em análise oportunamente explica o que vem a ser MOOC e suas características destacando que se trata de cursos online, com possibilidade dos desenvolvedores alcançarem um número massivo de pessoas, de forma que os estudantes podem ter acesso aos conteúdos no horário, tempo e local que tem disponível, tendo maior autonomia para determinar o prazo de término de seu curso. Basta ter um dispositivo com acesso a internet, assim pode-se dizer que MOOCs são ferramentas de suporte pedagógico e, a TIM Tec é um ambiente virtual, uma plataforma que favorece os processos de ensino e a aprendizagem (montagem de aulas, prático e fácil de atualizar). 
O estudo observa que se têm diferentes plataformas de ensino a distância - EaD, ou Ambientes Virtuais de Aprendizagem - AVA, que hospedam MOOCs tais como Coursera, edX, Eudacity, entre outas além da TIM Tec, contudo esta é uma iniciativa da empresa de telefonia TIM. O Instituto TIM Tec dispõe portanto de uma plataforma do tipo MOOC, com oferta de cursos livres, abertos e gratuitos alinhados ao Programa Nacional de Acesso ao Ensino Técnico e Emprego (PRONATEC), priorizando o uso de seu espaço para formação profissional.

A Plataforma TIM Tec é um ambiente virtual que hospeda os MOOCs. Nela além dos docentes poderem fazer uso, no desenvolvimento de cursos do tipo MOOC para aplicar aulas à distância, podem ainda fazer uso da plataforma junto ao ensino presencial (de forma hibrida), favorecendo a atualização rápida de conteúdo, a reposição de aulas; desenvolvimento de projeto pedagógico, atividades de reforço. Oferecendo assim diversificadas possibilidades de apoio pedagógico, através da conexão reutilização de conteúdos e motivação.

$\mathrm{Na}$ atualidade vive-se o conceito de conectivismo, onde as pessoas passam a maior parte do tempo conectadas à dispositivos, que por sua vez estão interconectados, de forma que a realidade se compõe de uma rede que compartilham entre si aplicativos, programas, ambientes e redes sociais, favorecendo acesso a conteúdos e a conexão entre pessoas de forma que podem ocorrer tanto de forma sincrônica como assíncrona. A plataforma TIM Tec, deixa clara a compreensão desta realidade, e têm recursos que facilitam esta interação (entre pessoas e aplicações, programas, ambientes, redes, entre outros) tendo com isto por propostas, ampliar as condições de formação docente; ampliar os espaços de aprendizagem para além da sala de aula (além dos muros da escola); proposta de envolver o aluno, usando tecnologias para aumentar o engajamento dos alunos, aproximá-los do professor (no sentido de que a distância física não seja um problema), a comunicação caracteriza-se como assíncrona e o docente tem condições de gerenciar turmas diferentes, e readequar conteúdos conforme demandas.

A proposta da TIM Tec é voltada predominantemente para o uso de micro vídeos, o que refere ao método de ensino denominado micro aprendizagem, onde há predomínio da linguagem informal, que aproxima o professor-instrutor do aluno em um contexto virtual, contudo respeita as diferentes características dos alunos (os estilos cognitivos, seu repertório 
de conhecimento, ritmo de aprendizagem) de forma que orienta a formulação dos vídeos curtos tanto para a produção do docente como do aluno (em formato de tarefa), onde a este é gerada a possibilidade de tornar-se protagonista de seu conhecimento, estes fatores atentam para o uso da metodologia de sala de aula invertida.

\section{REFERÊNCIAS}

ALMEIDA, D. S.; MARQUES, P. F. MOOCs: uma análise das experiências pioneiras no Brasil e Portugal - constatações e limitações. Portal ABED. Porto Alegre, abr. 2015. Disponível em: http://www.abed.org.br/congresso2or5/anais/pdf/BD_215.pdf Acesso em 27 jun. 2021.

ANDRADE, M. V. M. ; SILVEIRA, I. F. Panorama da Aplicação de Massive Open Oline Course (MOOC) no Ensino Superior: Desafios e Possibilidades. EaD em Foco, 6 (3), IoI-II4. 2016. https://eademfoco.cecierj.edu.br/index.php/Revista/article/view/392/220 Acesso em 20 mai. 202I.

BALBINO, V. S.; PINTO, S. C.; BRAZ, R. M. M..Uma visão do uso de MOOCs como ferramenta para capacitação e docentes de alunos com TEA. Revista Ensino Educação e Ciências Humanas, vol. 2I, n. 2, p.I8I-I90, 2020. Disponível em: https://www.researchgate.net/publication/34648645I_Uma_Visao_do_Uso_de_MOOCs_c omo_Ferramenta_de_Capacitacao_para_Docentes_de_Alunos_com_TEA Acesso em 24 jun. 2021.

BARIN, C., S. e BASTOS, F. P. da. Problematização dos MOOCs na atualidade: Potencialidades e Desafios. Revista Renote, v. II, n.3, 2013 Disponível em: https://seer.ufrgs.br/renote/article/view/44707/28546 Acesso em: 13 jul. 2021.

BASTOS, I. M. M. Desenho Pedagógico e Aprendizagem em MOOC: um estudo analítico sobre a qualidade dos Cursos em Plataformas Nacionais e Internacionais. 2016, p. I62. (Dissertação de Mestrado) Universidade Federal do Maranhão. Programa de Pós-graduação em Cultura e Sociedade. São Luís, 2016. Disponível em: https://tedebc.ufma.br/jspui/ bitstream/tede/152I/2/IsisMariaBastos.pdf Acesso em 27 jun. 202I.

BASTOS, R. C.; BIAGIOTTI, B. MOOCs: uma alternativa para democratização do ensino. Revista Renote, Novas tecnologias na Educação. vi2, n.I, 2014. Disponível em: https://seer.ufrgs.br/renote/article/view/50333/31417 Acesso em or jun. 2021.

FLATSCHART, F. MOOC e novas formas de aprendizagem. Plataforma do Instituto TIM Tec. 202I.

FURTADO, P. MOOC e novas formas de aprendizagem. Plataforma do Instituto TIM Tec. 202I. 
GÓMEZ, A. I. P. Educação na Era Digital. A escola educativa. Porto Alegre. Ed. Penso: 2015. E-book digital. Google Livros. Disponível em: https://books.google.com.br/books?hl=pt-

$\mathrm{BR} \& \mathrm{lr}=\& \mathrm{id}=\mathrm{nrEkBQAAQBAJ} \& o \mathrm{i}=\mathrm{fnd} \& \mathrm{pg}=\mathrm{PAI} \& \mathrm{dq}=\mathrm{Em}+$ plena+era + digital $+o$ s + docentes $+\mathrm{a}+\mathrm{educa} \% \mathrm{C}_{3} \% \mathrm{~A}_{7 \%} \mathrm{C}_{3} \% \mathrm{~A}_{30}$ \&ots=VHR4h35urF\&sig=zYBWjdjMmDHo5 $\mathrm{U}-$

CQPxkOprooA8\#v=onepage\&q=Em\%2oplena\%20era\%2odigital\%20os\%2odocentes\%20a\% 2oeduca\% $\mathrm{C}_{3} \% \mathrm{~A}_{7 \%} \mathrm{C}_{3 \%} \mathrm{~A} 30 \& \mathrm{f}=$ false Acesso em: 02 jun. 2021.

MENDES, A. R. MOOC e novas formas de aprendizagem. Plataforma do Instituto TIM Tec. 2021.

PUJOL, Leonardo. Coronavírus: menos aulas presenciais, mais EAD. Postagem em 12 mar. 2020. Disponível em: https://desafiosdaeducacao.grupoa.com.br/ead-alternativacoronavirus/ Acesso em: or set. 2020.

SÉRIE EDUCAR - Volume 44. Tecnologias Organização: Editora Poisson - Belo Horizonte M.G.: Poisson, 2020. Disponível em: https://www.poisson.com.br/livros/serie_ educar/volume44/Educar_vol44.pdf?fbclid=IwARIBSXjdjhuveKpVEM5F2wihzzFhp6Jco VYCo7ERdiSyTqhnz4pOafikjWA Acesso em 28 jun. 2021.

SILVA, P. G.; MARQUES, P. F. MOOC como possibilidade de Ensino e Aprendizagem em cultura digital. In TISE Congresso Internacional de Informática Educativa, Nuevas Ideas en Informatica Educativa TISE, vol.11, 232-239, Santiago Chile, 2015. Disponível em: http://www.tise.cl/volumenII/TISE2015/232-239.pdf Acesso em 27 jun. 202I.

SILVA, H. S. Revisão Sistemática sobre o uso de MOOCs no Brasil. (TCC Artigo) Especialista em Tecnologias da Informação e Comunicação Aplicadas à Educação. Universidade Federal de Santa Maria. Constantina RS, 2017. Disponível em: https://repositorio.ufsm.br/bitstream/handle/r/r2046/TCCE_TICAE_EaD_2017_SILVA_ HENRIQUE.pdf? sequence $=\mathrm{I} \&$ is Allowed $=\mathrm{y}$ Acesso em 27 jun. 2021.

TIM TEC, Portal do Instituto. Quem Somos. O instituto. Conheça nossa história. Portal Instituto Tim Org s/a.s/d 2020a. Disponível em: https://institutotim.org.br/quem-somos/ Acesso em: 24 jun. 2021. 